\title{
Contexto de trabalho e satisfação profissional de enfermeiros que atuam na Estratégia Saúde da Família
}

\author{
Context of work and professional satisfaction of nurses who work in \\ the Family Health Strategy
}

Maria Mônica de Oliveira', Dixis Figueroa Pedraza $\mathbf{1}^{\mathbf{1} 2}$

DOI: 10.1590/0103-1104201912209

RESUMO A pesquisa objetivou avaliar o contexto de trabalho e a satisfação profissional de enfermeiros que atuam na Estratégia Saúde da Família no estado da Paraíba, estabelecendo uma análise comparativa entre equipes convencionais e do Programa Mais Médicos (PMM). Trata-se de um estudo transversal realizado com 50 enfermeiros de 34 municípios representativos de todas as gerências regionais de saúde do estado. Obtiveram-se informações quanto ao perfil dos profissionais, estrutura das unidades de saúde, contexto de trabalho e satisfação profissional. Para o contexto de trabalho, utilizou-se a Escala de Avaliação do Contexto do Trabalho, enquanto para apreender a satisfação profissional, foram utilizadas perguntas adaptadas das dimensões satisfação e remuneração da metodologia Great Place to Work. Em sua totalidade, os domínios concernentes à estrutura das unidades de saúde, ao contexto de trabalho e à satisfação profissional auferiram avaliações satisfatórias. Entretanto, constataram-se deficiências, principalmente na organização do trabalho. Não houve diferenças ao se comparar equipes convencionais e do PMM. Correlações importantes e significativas foram observadas entre o contexto de trabalho e suas subdimensões com a satisfação profissional. Esta pesquisa evidencia condições críticas de organização do trabalho que influenciam negativamente a satisfação profissional, com problemas na gestão do processo de trabalho que focam o aumento da produtividade.

1 Universidade Estadual da Paraíba (UEPB) - Campina Grande (PB), Brasil. monicaenf17@gmail.com

2 Rede Nordeste de Formação em Saúde da Família (Renasf) Natal (RN), Brasil.
PALAVRAS-CHAVE Atenção Primária à Saúde. Estratégia Saúde da Família. Condições de trabalho. Satisfação no emprego.

ABSTRACT The research aimed to evaluate the work context and the professional satisfaction of nurses who work in the Family Health Strategy in the State of Paraíba establishing a comparative analysis between conventional teams and those of the More Doctors Program. This is a cross-sectional study carried out with 50 nurses from 34 municipalities representing all the regional health managements in the State. Information was obtained on the profile of professionals, structure of health units, work context, and professional satisfaction. The Work Context Assessment Scale was used for the context of work, while for the purpose of apprehending professional satisfaction, questions adapted from the satisfaction and remuneration dimensions of the Great Place to Work methodology were used. In their totality, the domains concerning the structure of the health units, the work context and the professional satisfaction have received satisfactory evaluations. However, there were deficiencies, mainly in the organization of work. There were no differences when comparing 
conventional and More Doctors Program teams. Important and significant correlations were observed between the work context and its sub-dimensions with job satisfaction. This research shows critical conditions of work organization that negatively influence professional satisfaction, with problems in the management of the work process that focus on increasing productivity.

KEYWORDS Primary Health Care. Family Health Strategy. Working conditions. Job satisfaction.

\section{Introdução}

A Atenção Primária à Saúde (APS) constitui-se elemento essencial de um processo continuado de cuida-do integral ao indivíduo, no contexto da família e da comunidade. Configura-se também como base e importante estratégia para a reorientação das práticas profissionais, da reorganização dos serviços e da ampliação da efetividade em todos os demais níveis dos sistemas de saúde ${ }^{1-4}$.

No Brasil, no intuito de ampliar a cobertura e o acesso à população aos serviços de saúde, a Estratégia Saúde da Família (ESF) estabelece-se como modelo de reordenação da atenção à saúde conforme os princípios do Sistema Único de Saúde (SUS) 1 .

Compreende-se que o modelo de assistência proposto na ESF requer condições estruturais mínimas para a execução das ações que ultrapassem o modelo biomédico e que contribuam para a equidade em saúde. A estrutura adequada, a organização das ações consoantes com os princípios do SUS e o suporte administrativo constituem pontos centrais na prestação dos serviços pelas equipes de saúde ${ }^{4,5}$.

Os sistemas de saúde devem oferecer serviços eficazes, seguros e eficientes ${ }^{6}$. Nesse contexto, para efetivação da qualidade da assistência oferecida ao usuário e da garantia da promoção à saúde, é fundamental que o ambiente de trabalho possibilite a integração da equipe multidisciplinar, como também favoreça qualidade de vida, motivação, satisfação e possibilidade de desenvolvimento ao profissional de saúde ${ }^{7,8}$. Nesse sentido, reitera-se que o serviço prestado pelas equipes de saúde depende proporcionalmente do suporte administrativo, da adequação de estrutura e da organização das ações em consonância com os princípios do SUS?.

As características do processo de trabalho no modelo da ESF têm implicações na satisfação/insatisfação dos profissionais de saúde, a qual condiciona diretamente a qualidade das ações em saúde direcionadas à população ${ }^{6,10}$. A combinação de fatores organizacionais, estruturais e financeiros, tais como dificuldades gerenciais, burocracia excessiva, sobrecarga de trabalho, precariedade das condições de trabalho, deficiências estruturais, falta de equipamentos adequados, baixos salários e ausência de uma política de educação continuada repercute consubstancialmente na insatisfação dos profissionais das equipes de saúde da família e coloca em discussão questões sobre a qualificação dos profissionais, a resolubilidade dos serviços e a necessidade de monitorar e avaliar a APS $\mathbf{2 , 5 , 6 , 1 1}$. Ainda, essas condições podem resultar em problemas de saúde nos próprios trabalhadores que prestam serviços de saúde ${ }^{11,12}$.

Os profissionais da APS constituem um enorme contingente de força de trabalho no sistema de saúde brasileiro; e a enfermagem está amplamente inserida nesta área de atuação. A ESF tem no enfermeiro um importante membro da equipe básica multidisciplinar. No que se refere ao contexto de trabalho, esse profissional destaca-se ao desempenhar papel de liderança ampliada, incorporando-se funções importantes em práticas gerenciais, 
assistenciais, e no entendimento dos fatores interferentes no processo saúde-doença, a fim de suprir as demandas populacionais ${ }^{8,13,14}$.

Considerando ainda que, para o fortalecimento da APS, torna-se primordial a utilização de mecanismos que permitam monitorar as condições relacionadas com o processo de trabalho ${ }^{9}$, o presente estudo tem como objetivo avaliar o contexto de trabalho e a satisfação profissional de enfermeiros que atuam na ESF no estado da Paraíba, estabelecendo uma análise comparativa entre equipes convencionais e do Programa Mais Médicos (PMM).

\section{Métodos}

Trata-se de um estudo transversal realizado com uma amostra de 50 enfermeiros, de um total de 62 previstos, que atuam em equipes da ESF, selecionada aleatoriamente, de 34 municípios com população inferior a 30 mil habitantes, representativos de todas as gerências regionais de saúde do estado da Paraíba.

Para a coleta de dados utilizou-se um questionário estruturado previamente testado que foi registrado no FormSUS (http://siteformsus.datasus.gov.br/FORMSUS/index.php), enviado via $e$-mail, com link de acesso, acompanhado de convite para participar da pesquisa, orientações, esclarecimentos e Termo de Consentimento Livre e Esclarecido (TCLE). Foram obtidas informações em quatro âmbitos: perfil dos profissionais (características demográficas, capacitação em APS, características trabalhistas), estrutura das unidades de saúde, contexto de trabalho e satisfação profissional.

Sexo, idade, convivência com companheiro(a) e número de filhos foram as características demográficas consideradas. A capacitação em APS baseou-se na informação sobre a realização de pós-graduação ou residência em saúde pública/saúde coletiva/saúde da família/medicina de família/saúde materno-infantil e de em algum curso ou treinamento em APS/ESF. As características trabalhistas foram descritas considerando o tempo de atuação na equipe de saúde atual e no tipo de vínculo.

A estrutura das unidades de saúde considerou três subdimensões: i. disponibilidade de recursos humanos (serviços ininterruptos do médico, enfermeiro e auxiliar ou técnico de enfermagem) e espaços físicos (recepção, sanitário e depósito, consultório próprio, sala de reuniões e atividades de educação, sala exclusiva para vacinação, sala exclusiva para curativos e observação, copa/cozinha); ii. disponibilidade de equipamentos, acessórios de informática/telefonia e insumos (geladeira, antropômetro, estadiômetro ou fita métrica, balança infantil, balança antropométrica, computador, acesso à internet, linha telefônica, Caderneta de Saúde da Criança, transporte); e iii. disponibilidade de vacinas, medicamentos essenciais e suplementos (vacinas do calendário básico de imunização da criança, medicamentos básicos indicados nos tratamentos das parasitoses mais frequentes, antibióticos básicos indicados nos tratamentos das doenças infecciosas mais frequentes, medicamentos para o tratamento das doenças do trato gastrointestinal, medicamentos inalatórios para a crise asmática, antitérmicos, anti-inflamatórios, sais de reidratação oral, sulfato ferroso, suplemento de Vitamina A). Atributos com respostas negativas receberam pontuação $=0,5$, e positivas, pontuação=0,1; podendo, assim, variar de 1 a 5 a avaliação de cada uma das subdimensões da estrutura. A avaliação geral da estrutura das unidades de saúde foi baseada na média das suas subdimensões.

O contexto de trabalho compreendeu informações de organização do trabalho, relações socioprofissionais e condições de trabalho, obtidas utilizando a Escala de Avaliação do Contexto do Trabalho (EACT), a qual forma parte do instrumento denominado Inventário sobre Trabalho e Risco de Adoecimento, validado previamente ${ }^{15}$. A EACT é uma escala do tipo Likert de cinco pontos (valor $1=$ nunca, $2=$ raramente, $3=$ às vezes, $4=$ frequentemente e $5=$ sempre) que se referem à frequência 
de ocorrência de determinados fenômenos, podendo variar de 1 a 5 cada atributo do contexto de trabalho. A avaliação de cada subdimensão teve por base a média dos seus atributos, bem como o contexto de trabalho derivou da média das três subdimensões.

Para apreender a satisfação profissional foram utilizadas perguntas adaptadas das dimensões satisfação e remuneração da metodologia Great Place to Work para Pesquisa de Clima Organizacional (Pesquisa de Clima Organizacional. Disponível em: http://www. greatplacetowork.com.br/pesquisa-e-benchmark/pesquisa-de-clima-organizacional.htm. Acesso em: 18 set 2018). Perguntou-se: 1. Você está satisfeito com suas atividades profissionais e considera que seu trabalho contribui para o sucesso da sua equipe? 2. Você indicaria sua unidade de saúde para um processo seletivo de um amigo ou conhecido? 3. Você indicaria sua unidade de saúde para alguém da sua família ou amigo utilizar? 4. Você considera justo seu salário? 5 . Você está satisfeito com a forma pela qual sua equipe contribui para a sociedade? As alternativas de respostas usadas foram 'sim' e 'não'. Cada resposta negativa recebeu pontuação=1,0, e positiva, pontuação= 0,2 ; podendo, assim, variar de $1 \mathrm{a}$ 5 a avaliação geral da satisfação profissional.

A classificação da estrutura, contexto de trabalho e satisfação profissional obedeceu às recomendações para o contexto de trabalho, considerando este o eixo central do presente estudo: acima de 3,7 (grave), entre 2,3 e 3,69 (crítica) e abaixo de 2,29 (satisfatória) ${ }^{15}$.

Os dados coletados foram organizados em planilhas eletrônicas e digitados em dupla entrada em um banco de dados customizado com verificações de consistência e restrições de intervalo. As inconsistências entre digitadores foram verificadas e retificadas por meio de nova consulta aos respectivos questionários.

Diferenças segundo o tipo de equipe (convencional ou do PMM) para as variáveis do perfil dos profissionais foram analisadas usando o teste de qui-quadrado ou Exato de Fisher. Foram calculadas as médias ( \pm DP) das pontuações relacionadas com a estrutura das unidades de saúde, contexto de trabalho e satisfação profissional, estabelecendo-se diferenças segundo o tipo de equipe por meio do teste t-student. Analisou-se a correlação das avaliações da estrutura das unidades de saúde e do contexto de trabalho com a satisfação profissional por meio do coeficiente de correlação de Pearson. A normalidade dos dados foi aferida por meio do teste de Kolmogorov-Smirnov. Em todas as análises, o nível de significância admitido foi de $5 \%$. Utilizou-se o software Stata v.12.0.

A pesquisa foi aprovada pelo Comitê de Ética em Pesquisa da Universidade Estadual da Paraíba, com o protocolo número 71609317.9.0000.5187. Todos os participantes da pesquisa assinaram o TCLE, como condição necessária à participação no estudo.

\section{Resultados}

De acordo com a tabela 1, a maioria dos enfermeiros entrevistados é do sexo feminino (88\%), está na faixa etária de idade entre 20 e 39 anos (70\%), convive com companheiro(a) (68\%) e não tem filhos (76\%). Quanto à capacitação em APS, 26 dos 50 enfermeiros relataram possuir pós-graduação ou residência em saúde pública/saúde coletiva/saúde da família/medicina de família/saúde materno-infantil, e 37 que já fizeram algum curso ou treinamento em APS/ESF. Com relação às características trabalhistas, constatou-se que menos da metade dos profissionais entrevistados atuam na equipe de saúde há mais de dois anos e que são concursados. Observa-se essa caracterização tanto nas equipes convencionais como nas do PMM e sem diferenças entres elas. 
Tabela 1. Características demográficas, da formação e trabalhistas de enfermeiros da ESF da Paraíba, Brasil, 2018

\begin{tabular}{|c|c|c|c|c|}
\hline \multirow[t]{2}{*}{ Variáveis } & $\begin{array}{r}\text { Total de equipes } \\
(\mathrm{N}=50)\end{array}$ & $\begin{array}{r}\text { Equipes } \\
\text { convencionais } \\
(\mathrm{N}=26)\end{array}$ & $\begin{array}{r}\text { Equipes do PMM } \\
\qquad(\mathrm{N}=24)\end{array}$ & p-valor \\
\hline & $\mathrm{n}$ & $\mathrm{n}$ & $\bar{n}$ & \\
\hline \multicolumn{5}{|l|}{ Características demográficas } \\
\hline Sexo & & & & 0,329 \\
\hline Feminino & 44 & 24 & 20 & \\
\hline Masculino & 6 & 2 & 4 & \\
\hline Idade & & & & 0,459 \\
\hline 20 a 39 & 35 & 17 & 18 & \\
\hline 40 a 49 & 15 & 9 & 6 & \\
\hline Convivência com companheiro(a) (sim) & 34 & 17 & 17 & 0,680 \\
\hline Filhos (sim) & 12 & 8 & 4 & 0,243 \\
\hline \multicolumn{5}{|l|}{ Características da formação } \\
\hline $\begin{array}{l}\text { Pós-graduação ou residência em saúde públi- } \\
\text { ca/saúde coletiva/saúde da família/medicina } \\
\text { de família/saúde materno-infantil (sim) }\end{array}$ & 26 & 14 & 12 & 0,786 \\
\hline Curso ou treinamento em APS/ESF (sim) & 37 & 18 & 19 & 0,424 \\
\hline \multicolumn{5}{|l|}{ Características trabalhistas } \\
\hline $\begin{array}{l}\text { Tempo de atuação na equipe de saúde atual } \\
\text { (dois anos ou mais) }\end{array}$ & 23 & 10 & 13 & 0,266 \\
\hline Tipo de vínculo (concursado) & 22 & 10 & 12 & 0,412 \\
\hline
\end{tabular}

Fonte: Elaboração própria.

PMM: Programa Mais Médicos; p-valor: nível de significância.

Os resultados apresentados na tabela 2, concernentes à avaliação da estrutura das unidades de saúde, do contexto de trabalho e da satisfação profissional, mostram que a estrutura foi avaliada como satisfatória tanto no total de equipes (média 1,63) quanto nas convencionais (média 1,60) e nas do PMM (média 1,66). Foram positivamente avaliadas as três subdimensões relacionadas com a estrutura das unidades de saúde, tanto nas equipes i. convencionais como as do ii. PMM: recursos humanos e espaços físicos (médias i. 1,60; ii. 1,65); equipamentos, acessórios de informática/telefonia e insumos (médias i. 1,66; ii. 2,01); e vacinas, medicamentos essenciais e suplementos (médias i. 1,55; ii. 1,33). A disponibilidade de linha telefônica foi o item com pior avaliação, seguido de acesso à internet. As equipes do PMM tiveram médias maiores do que as equipes convencionais nas variáveis serviços ininterruptos do enfermeiro e disponibilidade de transporte, bem como para a subdimensão disponibilidade de equipamentos, acessórios de informática/telefonia e insumos. 
Tabela 2. Avaliação da estrutura das unidades de saúde, do contexto de trabalho e da satisfação profissional por enfermeiros da ESF da Paraíba, Brasil, 2018

\begin{tabular}{|c|c|c|c|c|}
\hline \multirow[t]{3}{*}{ Variáveis } & \multicolumn{3}{|c|}{ Média (Desvio padrão) } & \multirow[t]{3}{*}{ p-valor } \\
\hline & \multirow{2}{*}{$\begin{array}{l}\text { Total de } \\
\text { equipes }\end{array}$} & \multirow{2}{*}{$\begin{array}{r}\text { Equipes } \\
\text { convencionais }\end{array}$} & \multirow{2}{*}{$\begin{array}{r}\text { Equipes do } \\
\text { PMM }\end{array}$} & \\
\hline & & & & \\
\hline \multicolumn{5}{|l|}{ ESTRUTURA } \\
\hline \multicolumn{5}{|l|}{ Recursos humanos e espaços físicos } \\
\hline Serviços ininterruptos do médico & $0,19(0,17)$ & $0,19(0,17)$ & $0,20(0,17)$ & 0,438 \\
\hline Serviços ininterruptos do enfermeiro & $0,17(0,15)$ & $0,13(0,10)$ & $0,21(0,18)$ & 0,024 \\
\hline Serviços ininterruptos do auxiliar ou técnico de enfermagem & $0,18(0,16)$ & $0,14(0,13)$ & $0,21(0,18)$ & 0,062 \\
\hline Espaço exclusivo para a recepção e espera & $0,11(0,07)$ & $0,13(0,10)$ & $0,10(1,42)$ & 0,913 \\
\hline Sanitário e depósito & $0,13(0,10)$ & $0,16(0,14)$ & $0,10(1,42)$ & 0,976 \\
\hline Consultório próprio (não compartilhado com outra equipe) & $0,10(0,05)$ & $0,11(0,07)$ & $0,11(1,42)$ & 0,829 \\
\hline Sala para realização de reuniões e atividades de educação & $0,22(0,18)$ & $0,23(0,19)$ & $0,21(0,18)$ & 0,656 \\
\hline Sala exclusiva para vacinação & $0,18(0,16)$ & $0,19(0,17)$ & $0,18(0,16)$ & 0,574 \\
\hline Sala exclusiva para curativos e observação & $0,15(0,14)$ & $0,16(0,14)$ & $0,15(0,13)$ & 0,612 \\
\hline Copa/cozinha & $0,14(0,13)$ & $0,13(0,10)$ & $0,16(0,15)$ & 0,169 \\
\hline Subtotal & $1,62(0,61)$ & $1,60(0,62)$ & $1,65(0,61)$ & 0,387 \\
\hline \multicolumn{5}{|l|}{ Equipamentos, acessórios de informática/telefonia e insumos } \\
\hline Geladeira & $0,19(0,17)$ & $0,19(0,17)$ & $0,20(0,17)$ & 0,438 \\
\hline Antropômetro & $0,14(0,13)$ & $0,14(0,13)$ & $0,15(0,13)$ & 0,459 \\
\hline Estadiômetro ou fita métrica & $0,10(0,05)$ & $0,11(0,07)$ & $0,10(1,42)$ & 0,829 \\
\hline Balança infantil & $0,11(0,07)$ & $0,10(0,00)$ & $0,10(1,42)$ & 0,500 \\
\hline Balança antropométrica & $0,11(0,07)$ & $0,10(0,00)$ & $0,13(0,11)$ & 0,069 \\
\hline Computador & $0,18(0,16)$ & $0,14(0,13)$ & $0,21(0,18)$ & 0,062 \\
\hline Acesso à internet & $0,23(0,19)$ & $0,20(0,18)$ & $0,26(0,20)$ & 0,140 \\
\hline Linha telefônica & $0,40(0,17)$ & $0,37(0,18)$ & $0,43(0,15)$ & 0,126 \\
\hline Caderneta de Saúde da Criança & $0,14(0,13)$ & $0,13(0,10)$ & $0,16(0,15)$ & 0,169 \\
\hline Transporte & $0,19(0,17)$ & $0,14(0,13)$ & $0,25(0,19)$ & 0,016 \\
\hline Subtotal & $1,83(0,54)$ & $1,66(0,55)$ & $2,01(0,48)$ & 0,010 \\
\hline \multicolumn{5}{|l|}{ Vacinas, medicamentos essenciais e suplementos } \\
\hline Vacinas do calendário básico de imunização da criança & $0,14(0,12)$ & $0,13(0,10)$ & $0,15(0,13)$ & 0,290 \\
\hline $\begin{array}{l}\text { Medicamentos básicos indicados nos tratamentos das parasi- } \\
\text { toses mais frequentes }\end{array}$ & $0,15(0,14)$ & $0,17(0,16)$ & $0,13(0,11)$ & 0,861 \\
\hline $\begin{array}{l}\text { Antibióticos básicos indicados nos tratamentos das doenças } \\
\text { infecciosas mais frequentes }\end{array}$ & $0,14(0,13)$ & $0,16(0,14)$ & $0,13(0,11)$ & 0,773 \\
\hline $\begin{array}{l}\text { Medicamentos para o tratamento das doenças do trato gas- } \\
\text { trointestinal }\end{array}$ & $0,14(0,13)$ & $0,16(0,14)$ & $0,13(0,11)$ & 0,773 \\
\hline Medicamentos inalatórios para a crise asmática & $0,14(0,12)$ & $0,14(0,13)$ & $0,13(0,11)$ & 0,643 \\
\hline Antitérmicos & $0,10(0,05)$ & $0,11(0,07)$ & $0,10(1,42)$ & 0,829 \\
\hline Anti-inflamatórios & $0,13(0,10)$ & $0,14(0,13)$ & $0,11(0,08)$ & 0,826 \\
\hline Sais de reidratação oral & $0,14(0,13)$ & $0,16(0.14)$ & $0,13(0,11)$ & 0,773 \\
\hline Sulfato ferroso & $0,16(0,14)$ & $0,19(0,17)$ & $0,13(0,11)$ & 0,919 \\
\hline
\end{tabular}


Tabela 2. (cont.)

\begin{tabular}{|c|c|c|c|c|}
\hline Suplemento de Vitamina A & $0,16(0,14)$ & $0,16(0,14)$ & $0,16(0,15)$ & 0,452 \\
\hline Subtotal & $1,44(0,73)$ & $1,55(0,86)$ & $1,33(0,57)$ & 0,851 \\
\hline Total - Estrutura & $1,63(0,45)$ & $1,60(0,51)$ & $1,66(0,38)$ & 0,318 \\
\hline \multicolumn{5}{|l|}{ CONTEXTO DE TRABALHO } \\
\hline \multicolumn{5}{|l|}{ Organização do trabalho } \\
\hline Falta tempo para realizar pausas de descanso no trabalho & $3,16(1,21)$ & $3,11(1,24)$ & $3,20(1,21)$ & 0,395 \\
\hline O ritmo do trabalho é excessivo & $3,12(1,13)$ & $3,13(1,13)$ & $3,10(1,15)$ & 0,582 \\
\hline As tarefas são cumpridas sob pressão de prazos & $2,68(1,44)$ & $2,65(1,49)$ & $2,70(1,42)$ & 0,448 \\
\hline Existe forte cobrança por resultados & $3,02(1,42)$ & $2,88(1,36)$ & $3,16(1,49)$ & 0,244 \\
\hline As normas para execução das tarefas são rígidas & $2,68(1,36)$ & $2,57(1,17)$ & $2,79(1,55)$ & 0,291 \\
\hline O número de pessoas é insuficiente para realizar as tarefas & $2,28(1,42)$ & $2,19(1,44)$ & $2,37(1,43)$ & 0,328 \\
\hline Os resultados esperados estão fora da realidade & $2,28(1,12)$ & $2,46(1,27)$ & $2,08(0,92)$ & 0,880 \\
\hline Existe fiscalização do desempenho & $3,58(1,26)$ & $3,53(1,27)$ & $3,62(1,27)$ & 0,405 \\
\hline Existe divisão entre quem planeja e quem executa & $2,68(1,42)$ & $2,50(1,30)$ & $2,87(1,54)$ & 0,178 \\
\hline As tarefas executadas sofrem descontinuidade & $2,38(0,98)$ & $2,42(0,98)$ & $2,33(1,00)$ & 0,624 \\
\hline Subtotal & $2,77(0,65)$ & $2,75(0,62)$ & $2,80(0,70)$ & 0,345 \\
\hline \multicolumn{5}{|l|}{ Condições de trabalho } \\
\hline As condições de trabalho são precárias & $2,12(1,18)$ & $1,92(1,12)$ & $2,33(1,23)$ & 0,113 \\
\hline O ambiente físico é desconfortável & $1,76(1,17)$ & $1,73(1,25)$ & $1,79(1,10)$ & 0,428 \\
\hline Existe muito barulho no ambiente de trabalho & $2,48(1,18)$ & $2,46(1,02)$ & $2,50(1,35)$ & 0,454 \\
\hline O mobiliário existente no local de trabalho é inadequado & $1,80(0,96)$ & $1,88(0,99)$ & $1,70(0,95)$ & 0,737 \\
\hline O posto de trabalho é inadequado para a realização das tarefas & $1,78(1,07)$ & $1,65(1,01)$ & $1,91(1,13)$ & 0,196 \\
\hline $\begin{array}{l}\text { As condições de trabalho oferecem riscos à segurança das } \\
\text { pessoas }\end{array}$ & $1,84(1,16)$ & $1,84(1,12)$ & $1,83(1,23)$ & 0,515 \\
\hline $\begin{array}{l}\text { Os instrumentos de trabalho são insuficientes para realizar as } \\
\text { tarefas }\end{array}$ & $1,94(1,01)$ & $2,00(1,05)$ & $1,87(0,99)$ & 0,665 \\
\hline $\begin{array}{l}\text { Os equipamentos necessários para realização das tarefas são } \\
\text { precários }\end{array}$ & $1,70(0,90)$ & $1,57(0,94)$ & $1,83(0,86)$ & 0,162 \\
\hline O espaço físico para realizar o trabalho é inadequado & $1,78(1,01)$ & $1,57(0,85)$ & $2,00(1,14)$ & 0,071 \\
\hline O material de consumo é insuficiente & $2,06(1,09)$ & $1,96(1,07)$ & $2,16(1,12)$ & 0,257 \\
\hline Subtotal & $1,92(0,71)$ & $1,86(0,72)$ & $1,99(0,71)$ & 0,256 \\
\hline \multicolumn{5}{|l|}{ Relações socioprofissionais } \\
\hline As tarefas são claramente definidas & $4,16(1,23)$ & $4,19(1,13)$ & $4,12(1,36)$ & 0,575 \\
\hline A autonomia é inexistente & $2,12(1,40)$ & $2,23(1,39)$ & $2,00(1,44)$ & 0,715 \\
\hline A distribuição das tarefas é injusta & $1,84(1,14)$ & $1,76(1,03)$ & $1,91(1,28)$ & 0,327 \\
\hline Os funcionários são excluídos das decisões & $1,76(1,02)$ & $1,84(1,04)$ & $1,66(1,00)$ & 0,729 \\
\hline Existem disputas profissionais no local de trabalho & $1,64(1,02)$ & $1,57(0,94)$ & $1,70(1,12)$ & 0,327 \\
\hline $\begin{array}{l}\text { As informações que precisa para executar suas tarefas são de } \\
\text { difícil acesso }\end{array}$ & $1,84(1,20)$ & $1,57(0,90)$ & $2,12(1,42)$ & 0,051 \\
\hline $\begin{array}{l}\text { Existem dificuldades na comunicação entre chefia e subordi- } \\
\text { nados }\end{array}$ & $1,58(0,88)$ & $1,38(0,57)$ & $1,79(1,10)$ & 0,053 \\
\hline Falta integração no ambiente de trabalho & $1,68(1,03)$ & $1,57(0,94)$ & $1,79(1,14)$ & 0,235 \\
\hline
\end{tabular}




\begin{tabular}{|c|c|c|c|c|}
\hline A comunicação entre os funcionários é insatisfatória & $1,74(1,06)$ & $1,76(1,03)$ & $1,70(1,12)$ & 0,578 \\
\hline Falta apoio das chefias para o seu desenvolvimento profissional & $2,24(1,30)$ & $2,07(1,26)$ & $2,41(1,34)$ & 0,181 \\
\hline Subtotal & $2,06(0,65)$ & $2,00(0,51)$ & $2,12(0,79)$ & 0,254 \\
\hline Total - Contexto de Trabalho & $2,23(0,54)$ & $2,20(0,50)$ & $2,28(0,59)$ & 0,237 \\
\hline \multicolumn{5}{|l|}{ SATISFAC̣ÃO } \\
\hline $\begin{array}{l}\text { Satisfação com as atividades profissionais, sentindo que seu } \\
\text { trabalho contribui para o sucesso da equipe }\end{array}$ & $0,26(0,21)$ & $0,23(0,15)$ & $0,30(0,27)$ & 0,134 \\
\hline $\begin{array}{l}\text { Indicaria a equipe de saúde para um processo seletivo de um } \\
\text { amigo ou conhecido }\end{array}$ & $0,23(0,15)$ & $0,23(0,15)$ & $0,23(0,16)$ & 0,477 \\
\hline $\begin{array}{l}\text { Indicaria a equipe de saúde para alguém da família ou amigo } \\
\text { utilizar }\end{array}$ & $0,21(0,11)$ & $0,20(0,00)$ & $0,23(0,16)$ & 0,151 \\
\hline Salário justo pelo trabalho & $0,84(0,32)$ & $0,78(0,36)$ & $0,90(0,27)$ & 0,105 \\
\hline $\begin{array}{l}\text { Satisfação com a forma pela qual a equipe de saúde contribui } \\
\text { para a comunidade/sociedade }\end{array}$ & $0,34(0,31)$ & $0,32(0,29)$ & $0,36(0,33)$ & 0,312 \\
\hline Total - Satisfação & $1,89(0,69)$ & $1,76(0,57)$ & $2,03(0,79)$ & 0,092 \\
\hline
\end{tabular}

Verificou-se que, na sua totalidade, o contexto de trabalho foi avaliado como satisfatório no total de equipes (média 2,23), nas convencionais (média 2,20) e nas do PMM (média 2,28). No que se refere à organização do trabalho, a falta de pessoal para a realização das tarefas foi avaliada criticamente nas equipes do PMM (média 2,35); enquanto nas equipes convencionais (média 2,19), teve avaliação positiva. O inverso foi observado para 'os resultados esperados estão fora da realidade', que foi avaliado positivamente nas equipes do PMM (média 2,08) e como crítico nas equipes convencionais (média 2,46). Avaliaram-se como críticos em ambos os tipos de equipes (i. convencional; ii. PMM) a existência de tempo para realizar pausas de descanso durante o trabalho (médias i. 3,11; ii. 3,20), o ritmo de trabalho (médias i. 3,13; ii. $3,10)$, a pressão em termos de prazos para a realização do trabalho (médias i. 2,65; ii. 2,70), a cobrança por resultados (médias i. 2,88; ii. $3,16)$ e a divisão de trabalho entre planejadores e executores (médias i. 2,50; ii. 2,87), caracterizadas como estratégias de gestão voltadas para o aumento da produtividade. Observa-se, ainda, que existem problemas na gestão do processo de trabalho; assim como foram avaliadas como críticas a rigidez das normas para a execução das tarefas (médias i. 2,57; ii. 2,79), a descontinuidade na execução destas (médias i. 2,42; ii. 2,33) e a fiscalização de desempenho no trabalho (médias i. 3,53; ii. 3,62 ). No geral, a organização do trabalho foi avaliada com situação crítica no total de equipes (média 2,77), nas equipes convencionais (média 2,75) e do PMM (média 2,80).

As condições de trabalho foram avaliadas como críticas, no que se refere à existência de barulho no ambiente de trabalho, no total de equipes (média 2,48), nas equipes convencionais (média 2,46) e nas equipes do PMM (média 2,50). A precariedade das condições de trabalho também foi avaliada como crítica nas equipes do PMM (média 2,33). Todavia, foram positivamente avaliados nas equipes convencionais e do PMM os outros atributos sobre as condições de trabalho. Em sua 
totalidade, essa subdimensão obteve avaliação positiva no total de equipes (média 1,92), nas equipes convencionais (média 1,86) e do PMM (média 1,99).

Foi avaliada como grave, nas relações socioprofissionais, a falta de clareza na definição das tarefas, no total de equipes (média 4,16), nas equipes convencionais (média 4,19) e do PMM (média 4,12). Verificou-se também que a falta de apoio das chefias para o desenvolvimento profissional foi avaliada como crítica nas equipes do PMM (média 2,41). Foram positivamente avaliados nas equipes convencionais e do PMM os outros atributos relacionados com esta subdimensão, a qual obteve avaliação positiva no total de equipes (média 2,06), nas equipes convencionais (média 2,00) e do PMM (média 2,12).

A satisfação profissional foi avaliada como satisfatória no total de equipes (média 1,89), nas convencionais (média 1,76) e nas do PMM (média 2,03). Dos itens considerados, a maior insatisfação foi em relação ao salário. Da mesma forma que para a estrutura e para o contexto de trabalho, não houve diferenças nas médias de satisfação ao se comparar equipes convencionais e do PMM.

As subdimensões do contexto de trabalho mostraram correlações importantes e significativas com a satisfação profissional tanto no total de equipes quanto nas convencionais e do PMM. A correlação mais forte foi para as relações socioprofissionais. Resultado similar foi encontrado quando considerado o contexto de trabalho como dimensão, que apresentou correlação de $0,670(p=0,0000)$ com a satisfação profissional para o total de equipes; $0,736(\mathrm{p}=0,0000)$ no caso dos profissionais das equipes convencionais e $0,623(p=0,001)$ em enfermeiros trabalhando inseridos nas equipes do PMM (tabela 3).

Tabela 3. Correlação entre os escores da avaliação da estrutura das unidades de saúde e do contexto de trabalho com a satisfação profissional de enfermeiros da ESF da Paraíba, Brasil, 2018

\begin{tabular}{|c|c|c|c|c|c|c|}
\hline \multirow[t]{4}{*}{ Subdimensão / Dimensão } & \multicolumn{6}{|c|}{ SATISFAC̣ÃO PROFISSIONAL } \\
\hline & \multirow{2}{*}{\multicolumn{2}{|c|}{ Total de equipes }} & \multirow{2}{*}{\multicolumn{2}{|c|}{$\begin{array}{r}\text { Equipes } \\
\text { convencionais }\end{array}$}} & \multirow{2}{*}{\multicolumn{2}{|c|}{ Equipes do PMM }} \\
\hline & & & & & & \\
\hline & $r$ & p-valor & $r$ & p-valor & $r$ & $\mathrm{p}$-valor \\
\hline Recursos humanos e espaços físicos & 0,178 & 0,215 & 0,304 & 0,132 & 0,107 & 0,525 \\
\hline $\begin{array}{l}\text { Equipamentos, acessórios de informática/telefonia e } \\
\text { insumos }\end{array}$ & 0,179 & 0,211 & 0,147 & 0,475 & 0,115 & 0,492 \\
\hline Vacinas, medicamentos essenciais e suplementos & 0,181 & 0,209 & 0,242 & 0,234 & 0,218 & 0,305 \\
\hline Estrutura & 0,249 & 0,080 & 0,308 & 0,125 & 0,196 & 0,352 \\
\hline Organização do trabalho & 0,463 & 0,0007 & 0,512 & 0,006 & 0,426 & 0,037 \\
\hline Condições de trabalho & 0,544 & 0,0000 & 0,613 & 0,0009 & 0,492 & 0,014 \\
\hline Relações socioprofissionais & 0,608 & 0,0000 & 0,654 & 0,0003 & 0,579 & 0,003 \\
\hline Contexto de Trabalho & 0,670 & 0,0000 & 0,736 & 0,0000 & 0,623 & 0,001 \\
\hline
\end{tabular}

Fonte: Elaboração própria.

PMM: Programa Mais Médicos; r: coeficiente de correlação de Pearson; p-valor: nível de significância. 


\section{Discussão}

Ao analisar o perfil dos enfermeiros deste estudo, quanto ao sexo, evidenciou-se uma amostra majoritariamente feminina. Essa caracterização reproduz o panorama histórico dessa profissão, exercida nos seus primórdios quase que exclusivamente por mulheres, além de confirmar a forte tendência de feminilização da força de trabalho na APS, comprovada em várias pesquisas $8, \mathbf{1 3 , 1 4 , 1 6 , 1 7}$. Quanto à faixa etária dos profissionais, percebe-se que, assim como o encontrado, outros autores declararam ser, na sua maioria, adultos jovens os profissionais que atuam na $\mathrm{APS}^{8,10,14}$. No que se refere à situação conjugal, estudo realizado no interior de Minas Gerais identificou que a maioria dos profissionais de enfermagem vinculados à ESF era casadas ou em união estável ${ }^{8}$, resultados semelhantes aos dados da presente pesquisa.

No presente estudo, apesar de ter sido observada discreta preponderância de profissionais que possuem pós-graduação ou residência em saúde pública/saúde coletiva/ saúde da família/medicina de família/saúde materno-infantil e que fizeram algum curso ou treinamento em APS/ESF, alguns deles ainda carecem dessa formação, o que pode comprometer o desempenho da APS, sobretudo no conjunto de atributos essenciais ${ }^{1,3,18}$. Assim, levando em consideração a diversidade de ações e conhecimento técnico específico que competem ao profissional de enfermagem ao atuar na ESF, torna-se fundamental que os serviços desenvolvam programas de educação permanente e continuada para todos os profissionais ${ }^{19}$. Nesse sentido, salienta-se que diversas estratégias têm sido formuladas e incentivadas pelo Ministério da Saúde para promover a capacitação profissional e qualificar a atenção à saúde, como a Política de Educação Permanente do SUS ${ }^{20}$. Enquanto, neste estudo, $52 \%$ e $74 \%$ dos enfermeiros possuíam pós-graduação ou residência e capacitação, relacionada com a APS, respectivamente, pesquisa realizada na região noroeste de Goiânia evidenciou que $71,4 \%$ dos enfermeiros tinham especialização e que $86,4 \%$ haviam participado em cursos $^{18}$. Essa disparidade pode estar relacionada com o fato de que é nos grandes centros urbanos que se localizam os principais polos de formação em saúde, possibilitando aos profissionais que atuam nessas cidades um maior acesso a cursos ${ }^{21}$.

Concernente às características trabalhistas, resultados similares aos encontrados neste estudo foram evidenciados anteriormente por autores que retrataram que uma minoria dos profissionais ingressa na ESF por meio de concurso público ${ }^{11,22}$ e tem vínculo de dois anos ou mais com a equipe ${ }^{16,22}$. Ressalta-se que esse cenário é prejudicial, pois o concurso ou processo seletivo públicos são apontados como a única maneira legal de desprecarização do trabalho no SUS; e a rotatividade de profissionais e a instabilidade dos contratos incidem negativamente no estabelecimento de vínculo com a população, o que pode prejudicar a efetividade da assistência e a continuidade do cuidado ${ }^{22}$.

Em consonância com os resultados positivos encontrados na avaliação da dimensão da estrutura das unidades de saúde no presente estudo, a literatura relata que municípios de pequeno porte apresentam melhores condições neste quesito ${ }^{\mathbf{2 3} 24}$. Coincidindo com os achados encontrados, outras pesquisas também destacaram como principais problemas de estrutura a carência em itens como linha telefônica, equipamentos de informática, acesso à internet e veículo para realizar atividades fora das unidades ${ }^{5,22}$. A insuficiência da disponibilidade de equipamentos e acesso à internet igualmente foi ressaltada com base em dados nacionais, destacando desigualdades regionais que apontam a região Nordeste entre as que apresentam os piores resultados ${ }^{4}$. Fragilidades desse tipo podem interferir negativamente na continuidade do atendimento e nos atributos da APS, bem como limitam o potencial dos serviços para a consolidação da ESF 3 ,5. Essas deficiências têm sido reconhecidas pelo Governo Federal, reforçando-se a necessidade de garantir boa estrutura das unidades de saúde como requisito para 
prover condições adequadas de trabalho e de práticas profissionais, com ações como a construção ou reforma de unidades básicas de saúde (Requalifica UBS) ${ }^{25-27}$ e de um plano nacional para instalar internet banda larga em 12 mil unidades (Plano Nacional Banda Larga. Disponível em: http://dab.saude.gov.br/ portaldab/esus.php? conteudo=banda_larga. Acesso em: 3 dez 2018).

Estudos similares realizados com profissionais de enfermagem vinculados à APS em municípios do interior de Minas Gerais ${ }^{8} \mathrm{e}$ do Rio Grande do Sul28 verificaram que a organização do trabalho foi a dimensão do contexto de trabalho com pior avaliação ${ }^{8}$, o que ficou corroborado nesse estudo. Diante desses dados, sinalizam-se táticas de gestão com ênfase na produção, ou seja, apontam para a capacidade de o profissional produzir com rendimento, com base em situações de cobrança por resultados, ritmo de trabalho excessivo e pressão que caracterizam modelos antidemocráticos de gestão do trabalho ${ }^{8}$. Tais práticas desestimulam os profissionais e apontam para a necessidade de estratégias que promovam o bem-estar geral e do trabalho, essencial para um processo produtivo eficaz ${ }^{\mathbf{8}, 29}$.

A subdimensão condições de trabalho foi avaliada positivamente pelos profissionais que participaram desta pesquisa, com média $1,92 \pm 0,71$, correspondente a um cenário satisfatório, que indica situação melhor à encontrada em um município mineiro cuja média $2,75 \pm 0,82$ foi ilustrativa de um cenário crítico ${ }^{8}$. A avaliação negativa para a existência de barulho no ambiente de trabalho ${ }^{\mathbf{2 8}}$ e para a precariedade das condições de trabalho, por exemplo, cadeiras e mesas quebradas, ambientes pouco iluminados, salas interditadas ${ }^{11}$, encontra respaldo em observações anteriores da ESF em outras localidades do País. Uma pesquisa de revisão sistemática centrada no contexto de trabalho dos profissionais de enfermagem no Chile também apontou, entre outras características, precárias condições laborais ${ }^{30}$. Oferecer aos profissionais de saúde boas condições de trabalho é crucial para o exercício pleno das atividades e adequada carga de trabalho, pois possibilita que o conhecimento e a experiência sejam devidamente aplicados ${ }^{\mathbf{1 4}, 17}$. Além disso, sugere-se sua importância em relação ao cansaço e ao estresse profissionais ${ }^{11}$. Nessa conjuntura, entende-se que as condições de trabalho devem possibilitar a adaptação e o acolhimento do indivíduo como pessoa e profissional, e, assim, proporcionar um incremento efetivo à produtividade? ${ }^{7}$. Deve ser ressaltado que, em específico, os achados do presente estudo apresentam coerência nas avaliações positivas de estrutura e condições de trabalho, uma vez que esta última característica se refere à qualidade do ambiente de trabalho, equipamentos e materiais disponíveis para realizar as atividades laborais.

As relações socioprofissionais nas equipes de ESF no estudo obtiveram avaliação dominantemente positiva, melhor à constatada em outra realidade do País ${ }^{8}$. Entretanto, a clareza na definição das atividades e o apoio da chefia para o desenvolvimento profissional não tiveram avaliações tão positivas, o que também diverge de resultados prévios que apontaram contextos favoráveis para esses aspectos em unidades básicas de um município do interior do estado do Rio Grande do Sul28 e de 271 municípios catarinenses ${ }^{9}$ respectivamente. Em relação ao suporte oferecido pelas instâncias superiores, cabe destacar que vários estudos também relataram avaliações negativas 21,28,31. É conveniente ressaltar que podem contribuir positivamente para os aspectos organizativos relacionados com a acepção de tarefas; com a disponibilidade de protocolos e de programas de assistência ${ }^{\mathbf{2 8}}$; com a interação dos profissionais em cargos de direção com aqueles responsáveis pela prestação dos serviços de saúde, por meio da qualificação de gestores em ações de educação permanente 18,20,21, e com as relações entre os profissionais com trabalho cooperativo, afetuoso e em equipe ${ }^{\mathbf{8}, 32}$.

O nível de satisfação positivo dos profissionais de saúde da APS do presente estudo assemelha-se aos resultados encontrados tanto em pesquisas prévias desenvolvidas no Brasil14,33 quanto em outros países ${ }^{6,34}$. Apesar 
de algumas condições do trabalho não serem tão favoráveis, segundo a avaliação dos profissionais, o grau de satisfação positivo destes pode envolver aspectos relacionados com o comprometimento com as responsabilidades laborais e com a afinidade com a profissão, capazes de gerar perspectivas de enfrentamento mesmo diante de contextos adversos ${ }^{17,32}$, os quais ficaram fora do alcance deste estudo. Contudo, a insatisfação com a remuneração vem sendo destacada na literatura, inclusive entre os profissionais de enfermagem ${ }^{\mathbf{8}, 12,13,17,31}$, confirmando-se também em revisão literária recente ${ }^{35}$ e na atual pesquisa. A satisfação dos profissionais de saúde é importante para a continuidade e melhoria dos serviços prestados, responsivos às necessidades de saúde da população, bem como ao contentamento dos usuários com o atendimento recebido ${ }^{6,34}$.

No geral, na presente pesquisa, não foram verificadas diferenças de estrutura, contexto de trabalho e satisfação profissional ao se comparar equipes convencionais e do PMM. Até onde é conhecimento dos autores, este é primeiro estudo com tais análises. Entretanto, outros pesquisadores revelaram, de forma semelhante, que não foram observadas diferenças em aspectos de estrutura, processo e resultados, auferidos por meio do Primary Care Assessment Tool, ao avaliar a assistência oferecida por equipes convencionais e do PMM em município do estado do Paraná ${ }^{2}$. Assim, por meio desses resultados é possível inferir que as condições de trabalho permanecem questionáveis tanto nas equipes de saúde convencionais quanto nas do PMM, apesar de o Programa representar a estratégia de maior êxito no Brasil para provimento de médicos e ter como um dos seus eixos a estruturação das unidades básicas de saúde ${ }^{36}$.

As correlações identificadas neste estudo entre o contexto de trabalho, e suas subdimensões, com a satisfação profissional assemelham-se aos achados de outras pesquisas ${ }^{6,32,34}$. Essas relações encontram base na influência das condições laborais, e do processo de organização e gestão na satisfação no trabalho ${ }^{12,14,17}$. Nesse contexto, a satisfação profissional pode ser favorecida por meio de melhorias em aspectos específicos do trabalho (treinamento, remuneração, carga laboral), de gestão (autonomia, reconhecimento, oportunidade de desenvolvimento) e nas relações interpessoais $6,34,35$.

O uso de questionário eletrônico nesta pesquisa possibilitou a obtenção de dados a baixo custo e com uniformidade da mensuração. $\mathrm{O}$ índice de resposta de 80,6\% foi similar ao de estudo desenvolvido com profissionais da APS em Navarra, Espanha, de 77,5\% ${ }^{6}$. Considerando que estudos similares dificilmente superam os $50 \%$ de participação $0^{6}$, a taxa de adesão obtida foi satisfatória e garante consistência interna dos resultados. Entre as ações que foram implementadas na tentativa de elevar o índice de resposta, algumas podem ser destacadas: identificação dos responsáveis pela pesquisa, explicações claras sobre a pesquisa e sua relevância, utilização de um questionário objetivo e sintético, elaboração do questionário em formato agradável, remissão do questionário várias vezes de forma personalizada, gestão na entrega e recolhida do questionário, contato contínuo e uso de formas diversas de comunicação solicitando a cooperação.

Como possível limitação, deve ser considerada a subjetividade dos instrumentos de avaliação do contexto de trabalho e da satisfação profissional. Destaca-se, ainda, a possibilidade de viés de informação, decorrente das respostas obtidas exclusivamente de profissionais de saúde.

\section{Conclusões}

Esta pesquisa evidencia, na percepção de enfermeiros, avaliações satisfatórias para a estrutura das unidades de saúde, o contexto de trabalho e a satisfação profissional, incluindo aspectos importantes da qualidade dos serviços de saúde que devem ser fortalecidos e ampliados. Entretanto, destacaram-se condições críticas de organização do trabalho que influenciam negativamente a satisfação profissional, com problemas na gestão do 
processo de trabalho que focam o aumento da produtividade e podem repercutir negativamente no desenvolvimento das atividades laborais dos profissionais de enfermagem que atuam na ESF. Essa conjuntura aponta a necessidade de adoção de modelos de gestão mais democráticos que utilizem estratégias de promoção do bem-estar do trabalho que estimulem os profissionais e contribuam com eficácia para o processo produtivo. Os achados mostrados envolvem questões subjetivas dos enfermeiros que podem não representar a realidade de outros profissionais de saúde.

\section{Colaboradores}

Oliveira MM (0000-0002-2775-4318)* contribuiu para a análise e interpretação dos dados; elaboração do rascunho e revisão crítica do conteúdo; e aprovação da versão final do manuscrito. Figueroa Pedraza D (0000-00025394-828X)* contribuiu para a concepção e planejamento, análise e interpretação dos dados; elaboração do rascunho e revisão crítica do conteúdo; e aprovação da versão final do manuscrito.

\section{Referências}

1. Lima EFA, Sousa AI, Leite FMC, et al. Avaliação da Estratégia Saúde da Família na perspectiva dos profissionais de saúde. Esc. Anna Nery Rev. Enferm. 2016; 20(2):275-280.

2. Carrer A, Toso BRGO, Guimarães ATB, et al. Efetividade da Estratégia Saúde da Família em unidades com e sem Programa Mais Médicos em município no oeste do Paraná, Brasil. Ciên. Saúde Colet. 2016; 21(9):2849-2860.

3. Turci MA, Lima-Costa MF, Macinko J. Influência de fatores estruturais e organizacionais no desempenho da atenção primária à saúde em Belo Horizonte, Minas Gerais, Brasil, na avaliação de gestores e enfermeiros. Cad. Saúde Pública. 2015; 31(9):1941-1952.

4. Bousquat A, Giovanella L, Fausto MCR, et al. Tipologia da estrutura das unidades básicas de saúde bra- sileiras: os 5 R. Cad. Saúde Pública. 2017; 33(8):1-14.

5. Moreira KS, Lima CA, Vieira MA, et al. Avaliação da infraestrutura das unidades de saúde da família e equipamentos para ações na Atenção Básica. Cogitare enferm. 2017; 22(2):1-10.

6. Pérez-Ciordia I, Guillén-Grima F, Brugos A, et al. Satisfacción laboral y factores de mejora en profesionales de atención primaria. An. sist. sanit. Navar. 2013; 36(2):253-262.

7. Chaves JA, Guimarães MGV. Equipe de enfermagem: análise do clima organizacional no centro cirúrgico do hospital universitário Getúlio Vargas. Somanlu. 2015; 15(2):78-98.

8. Marques ALN, Guimarães MBF, Duarte JMG, et al. Qualidade de vida e contexto de trabalho de profis-
*Orcid (Open Researcher and Contributor ID). 
sionais de enfermagem da Estratégia Saúde da Família. Rev. RENE. 2015; 16(5):672-681.

9. Fontana KC, Lacerda JT, Machado PMO. O processo de trabalho na Atenção Básica à saúde: avaliação da gestão. Saúde debate. 2016; 40(110):64-80.

10. Trindade LL, Pires DEP, Amestoy SC, et al. Trabalho na Estratégia da Saúde da Família: implicações nas cargas de trabalho de seus profissionais. Cogitare enferm. 2014; 19(3):528-535.

11. Maciel RHMO, Santos JBF, Rodrigues RL. Condições de trabalho dos trabalhadores da saúde: um enfoque sobre os técnicos e auxiliares de nível médio. Rev. bras. saúde ocup. 2015; 40(131):75-87.

12. Forte ECN, Pires DEP. Enfermeiras na atenção básica: entre a satisfação e a insatisfação no trabalho. Trab. educ. saúde. 2017; 15(3):709-724.

13. Figueroa Pedraza D, Queiroz D, Sales MC, et al. Caracterização do trabalho de enfermeiros e profissionais do Núcleo de Apoio à Saúde da Família na Atenção Primária. ABCS health sci. 2018; 43(2):77-83.

14. Tambasco LP. A satisfação no trabalho da equipe multiprofissional que atua na Atenção Primária à Saúde. Saúde debate. 2017; 41(esp):140-151.

15. Mendes AM. Psicodinâmica do trabalho: teoria método e pesquisas. São Paulo: Casa do Psicólogo; 2007.

16. Ferreira Neto JL, Oliveira GL, Viana NO, et al. Integralidade, condições de oferta de serviços e processo de trabalho de Equipes de Saúde da Família em Belo Horizonte. Saúde debate. 2016; 40(111):179-192.

17. Pires DEP, Machado RR, Soratto J, et al. Cargas de trabalho da enfermagem na saúde da família: implicações no acesso universal. Rev. Latino-Am. Enferm. 2016; 24 (esp):1-9.

18. Oliveira MPR, Menezes IHCF, Sousa LM, et al. Formação e qualificação de profissionais de saúde: fatores associados à qualidade da Atenção Primária. Rev. bras. educ. med. 2016; 40(4):547-559.
19. Ferreira SRS, Périco LAD, Dias VRFG. A complexidade do trabalho do enfermeiro na Atenção Primária à Saúde. Rev. bras. enferm. 2018; 71(supl1):752-757.

20. Cavalcanti PCS, Oliveira Neto, AV, Sousa MF. Quais são os desafios para a qualificação da Atenção Básica na visão dos gestores municipais? Saúde debate. 2015; 39(105):323-336

21. Mattos LB, Dahmer A, Magalhães CR. Contribuição do curso de especialização em Atenção Primária à Saúde à prática de profissionais da saúde. ABCS Health Sci. 2015; 40(3):184-189.

22. Alvarenga EC, Oliveira PTR, Pinheiro HHC, et al. Condições de trabalho de Equipes de Saúde da Família do Pará. Rev. NUFEN. 2018; 10(1):58-72.

23. Pimentel FC, Albuquerque PC, Souza WV. A Estratégia Saúde da Família no estado de Pernambuco: avaliação da estrutura das equipes por porte populacional. Saúde debate. 2015; 39(104):88-101.

24. Cardoso AVL, Chain APN, Mendes RIP, et al. Avaliação da gestão da Estratégia Saúde da Família por meio do instrumento Avaliação para Melhoria da Qualidade em municípios de Minas Gerais, Brasil. Ciênc. Saúde Colet. 2015; 20(4):267-284.

25. Brasil. Ministério da Saúde. Gabinete do Ministro. Portaria $n^{\circ} 339$, de 4 de março de 2013. Redefine o Componente Ampliação do Programa de Requalificação de Unidades Básicas de Saúde (UBS). Diário Oficial da União. 5 Mar 2013.

26. Brasil. Ministério da Saúde. Gabinete do Ministro. Portaria $\mathrm{n}^{\circ} 340$, de 4 de março de 2013. Redefine o Componente Construção do Programa de Requalificação de Unidades Básicas de Saúde (UBS). Diário Oficial da União. 5 Mar 2013.

27. Brasil. Ministério da Saúde. Gabinete do Ministro. Portaria ${ }^{\circ}$ 341, de 4 de março de 2013. Redefine o Componente Reforma do Programa de Requalificação de Unidades Básicas de Saúde (UBS). Diário Oficial da União. 5 Mar 2013. 
28. Maissiat GS, Laurtet L, Pai DD, et al. Contexto de trabalho, prazer e sofrimento na atenção básica em saúde. Rev. gaúcha enferm. 2015; 36(2):42-49.

29. Sulti ADC, Lima RCD, Freitas PSS, et al. O discurso dos gestores da Estratégia Saúde da Família sobre a tomada de decisão na gestão em saúde: desafio para o Sistema Único de Saúde. Saúde debate. 2015; 39(104):172-182.

30. Canales-Vergara M, Valenzuela-Suazo S, Paravic-Klijn T. Condiciones de trabajo de los profesionales de enfermería en Chile. Enferm. univ. 2016; 13(3):178186.

31. Soratto J, Pires DEP, Trindade LL, et al. Insatisfação no trabalho de profissionais da saúde na estratégia saúde da família. Texto \& contexto enferm. 2017; 26(3):1-11

32. Soratto J, Fernandes SC, Martins CF, et al. Aspectos geradores de satisfação e insatisfação dos profissionais da estratégia saúde da família de um município de pequeno porte da região sul do Brasil. Rev. CEFAC. 2018; 20(1):69-78.
33. Lorenz VR, Guirardello EB. O ambiente da prática profissional e Burnout em enfermeiros na atenção básica. Rev. Latino-Am. Enfermagem. 2014; 22(6):926933.

34. Dixit J, Goel S, Sharma V. A comparative study on the level of satisfaction among regular and contractual health-care workers in a Northern city of India. J. Family Med. Prim. Care. 2017; 6(2):416-423.

35. Halcomb E, Smyth E, Mcnnes S. Job satisfaction and career intentions of registered nurses in primary health care: an integrative review. BMC Fam. Pract. 2018; 19(1):136-149.

36. Mourão Netto JJ, Rodrigues ARM, Aragão OC, et al. Programa Mais Médicos e suas contribuições para a saúde no Brasil: revisão integrativa. Rev. panam. salud pública. 2018; 42(e):1-7.

Recebido em 02/01/2019

Aprovado em 10/05/2019

Conflito de interesses: inexistente

Suporte financeiro: não houve 